\title{
Primary ovarian insufficiency in a female with phosphomannomutase-2 gene (PMM2) mutations for congenital disorder of glycosylation
}

\author{
Yohei Masunaga $^{1)}$ *, Mie Mochizuki ${ }^{2}$ *, Machiko Kadoya ${ }^{3)}$, Yoshinao Wada ${ }^{3)}$, Nobuhiko Okamoto ${ }^{3)}$, \\ Maki Fukami ${ }^{4}$, Fumiko Kato ${ }^{1)}$, Hirotomo Saitsu ${ }^{5)}$ and Tsutomu Ogata ${ }^{1)}$ \\ 1) Department of Pediatrics, Hamamatsu University School of Medicine, Hamamatsu 431-3192, Japan \\ 2) Department of Pediatrics, University of Yamanashi, Faculty of Medicine, Chuou 409-3898, Japan \\ 3) Department of Molecular Medicine, Osaka Women's and Children's Hospital, Izumi 594-1101, Japan \\ 4) Department of Molecular Endocrinology, National Research Institute for Child Health and Development, Tokyo 157-8535, Japan \\ ${ }^{5)}$ Department of Biochemistry, Hamamatsu University School of Medicine, Hamamatsu 431-3192, Japan
}

\begin{abstract}
Primary ovarian insufficiency (POI) is a highly heterogeneous condition, and its underlying causes remain to be clarified in a large fraction of patients. Congenital disorders of glycosylation (CDG) are multisystem diseases caused by mutations of a number of genes involved in N-glycosylation or O-glycosylation, and the most frequent form is PMM2-CDG (alias, CDG-Ia) resulting from biallelic mutations in PMM2 encoding phosphomannomutase-2 involved in N-glycosylation. Here, we examined a 46,XX Japanese female with syndromic POI accompanied by an undetectable level of serum antiMüllerian hormone (AMH). Whole exome sequencing identified biallelic pathogenic mutations of PMM2 (a novel c. 34G $>$ C:p.(Asp12His) of maternal origin and a recurrent c.310C $>$ G:p.(Leu104Val) of paternal origin) (NM_000303.3), and Nglycosylation studies detected asialotransferrin and disialotransferrin characteristic of PMM2-CDG, in addition to normally glycosylated tetrasialotransferrin. Clinical assessment showed cerebellar hypotrophy, which is a fairly characteristic and highly prevalent feature in PMM2-CDG, together with multiple non-specific features reported in PMM2-CDG such as characteristic face, intellectual disability, skeletal abnormalities, and low blood antithrombin III value. These results including the undetectable level of serum AMH, in conjunction with previously reported findings suggestive of the critical role of glycosylation in oocyte development and function, imply that PMM2-CDG almost invariably leads to POI primarily because of the defective oogenesis and/or oocyte-dependent early folliculogenesis rather than the compromised bioactivity of FSH/LH with defective glycosylation. Thus, it is recommended to examine PMM2 in patients with syndromic POI, especially in those with cerebellar ataxia/hypotrophy.
\end{abstract}

Key words: Primary ovarian insufficiency, Congenital disorder of glycosylation, Phosphomannomutase-2

PRIMARY OVARIAN INSUFFICIENCY (POI) is defined as primary or secondary amenorrhea with hypergonadotropic (FSH $\geq 40 \quad \mathrm{IU} / \mathrm{L})$ hypoestrogenism in women under 40 years of age $[1,2]$. It occurs as a nonsyndromic form or as a syndromic form associated with

Submitted Nov. 7, 2020; Accepted Dec. 17, 2020 as EJ20-0706 Released online in J-STAGE as advance publication Feb. 11, 2021 Correspondence to: Tsutomu Ogata, Department of Pediatrics, Hamamatsu University School of Medicine, 1-20-1 Handayama, Higashi-ku, Hamamatsu, 431-3192, Japan.

E-mail: tomogata@hama-med.ac.jp

* Contributed equally to this work.

Abbreviations: $\mathrm{AMH}$, anti-Müllerian hormone; $\mathrm{CDG}$, congenital disorders of glycosylation; ESI-MS, electrospray ionization mass spectrometry; MRI, magnetic resonance imaging; PMM2, phosphomannomutase-2; POI, primary ovarian insufficiency; and WES, whole exome sequencing. extragenital features $[1,2]$. The underlying causes of POI are variable, including autoimmune, chromosomal, and gene abnormalities [1, 2]. Recently, multiple causative genes for POI have been identified by advanced molecular technologies such as next generation sequencing [2], although underlying causes still remain to be clarified in a large fraction of patients.

Congenital disorders of glycosylation (CDG) are multisystem diseases caused by mutations of a number of genes involved in $\mathrm{N}$-glycosylation or O-glycosylation [3]. N-glycans are linked to the amide group of asparagine, whereas O-glycans are linked to the hydroxyl group of serine or threonine. The most frequent form is PMM2-CDG (alias, CDG-Ia) resulting from biallelic mutations in $P M M 2$ encoding phosphomannomutase-2 involved in N-glycosylation [4-6]. PMM2 consists of 
eight exons and encodes 246 amino acids, and PMM2 catalyzes the isomerization of mannose 6-phosphate to mannose 1-phosphate [4]. PMM2-CDG is associated with a wide range of clinical features, including characteristic face, growth failure, intellectual disability, cerebellar hypotrophy, retinitis pigmentosa, peripheral neuropathy, skeletal abnormalities, and coagulation abnormalities [5, 6]. Notably, PMM2-CDG is also almost invariably accompanied by POI $[5,6]$. However, underlying mechanism(s) leading to POI remains largely unknown.

Here, we report a female with syndromic POI in whom PMM2 mutations were identified by whole exome sequencing (WES), and discuss the underlying mechanism(s) leading to POI.

\section{Case Presentation}

This Japanese female was born at 38 weeks of gestation, with a birth length of $45.5 \mathrm{~cm}(-1.5 \mathrm{SD})$ and a birth weight of $2.69 \mathrm{~kg}(-0.6 \mathrm{SD})$. She showed hypotonia and failure to thrive in infancy, and began to walk without support at $26 / 12$ years of age. She had status epilepticus with abnormal electroencephalograms at $63 / 12$ years of age. Thus, she was treated with sodium valproate until electroencephalograms became apparently normal at 15 years of age. She manifested moderate intellectual disability, and was enrolled in a special junior school for such children. Her stature remained between -1 SD and -2 SD until 10 years of age.

At 11 10/12 years of age, she was found to lack pubertal growth spurt and secondary sexual development when she came to our hospital due to common cold. She became short for her age with a height of $130.2 \mathrm{~cm}(-2.9$ SD) and a weight of $25.6 \mathrm{~kg}(-1.9 \mathrm{SD})$. Her breast development was at Tanner stage 1 , and her external genitalia were infantile. Physical examination at that time showed hypertelorism, asymmetric eye fissures, left internal strabismus, thin upper lips, well developed mandible, retracted nipples, kyphoscoliosis, and elongated slender fingers. There were no Turner-like external somatic features such as webbed neck and cubitus valgus. She exhibited ataxic gait and poor fine movement, but had no involuntary movement. Her visual, acoustic, and olfactory acuities were apparently normal. Endocrine studies showed severe hypergonadotropic hypogonadism, mild hyperprolactinemia, moderate hyperthyroglobulinemia, and apparently normal blood GH/IGF-1, ACTH/cortisol, TSH/T4, and glucose/HbAlc/insulin values (Table 1). Chromosome analysis showed a 46,XX karyotype. Her bone age was 8 10/12 years. Brain magnetic resonance imaging (MRI) delineated cerebellar hypotrophy (Fig. 1A), and abdominal MRI showed rudimentary uterus and failed to detect ovaries (Fig. 1B). Estrogen replacement therapy enlarged the uterine size, and subsequent cyclic estrogen-progestin therapy successfully induced menses from $159 / 12$ years of age. She also manifested mild antibody-negative hypothyroidism accompanied by overt hyperthyroglobulinemia and mild goiter with cystic masses from late teens (Table 1 ), and received $l$-thyroxin ( $25 \mu \mathrm{g}$ /day) from $197 / 12$ years of age. At 23 8/12 years of age shortly after the diagnosis of PMM2-CDG, we screened for clinical findings reported in PMM2-CDG $[5,6]$, revealing a low blood antithrombin III value of $51 \%$ (reference range, $80-130 \%$ ). We also found an unmeasurable level of blood anti-Müllerian hormone $(\mathrm{AMH})$ (Table 1). At that time, she measured $152.5 \mathrm{~cm}$ (-1.1 SD) and weighed $47.5 \mathrm{~kg}(-0.7 \mathrm{SD})$.

The 65-year-old father and the 62-year-old mother were non-consanguineous and healthy. The mother had menarche at 12 years of age (reference data, 9.75-14.75 years) followed by regular menses until 46 years of age, and had menopause at 47 years of age (45-56 years). The mother did not have a history of other pregnancies or fetal abortions.

\section{Molecular Studies}

This study was approved by the Institutional Review Board Committee at Hamamatsu University School of Medicine, and was performed using leukocyte genomic DNA samples of the patient and the parents after obtaining written informed consent. WES was carried out by the previously described methods [7], using Human GRCh37/h19 as the reference genome. In short, WES was performed with SureSelect Human All Exon V6 (Agilent Technologies), and captured libraries were sequenced by NextSeq 500 (Illumina) with 150 bp paired-end reads.

Consequently, we identified compound heterozygous missense mutations of PMM2, i.e., c.34G $>\mathrm{C}: \mathrm{p}$. (Asp12His) of maternal origin and c.310C $>\mathrm{G}: \mathrm{p}$. (Leu104Val) of paternal origin (NM_000303.3) (Table 2). These mutations were confirmed by Sanger sequencing (Fig. 2A). They were almost completely absent from the public and in-house databases shown in Table 2, and were assessed to have high pathogenicity by several methods shown in Table 2. According to the ACMG Standards and Guidelines, the p.(Asp12His) was evaluated as "likely pathogenic" (positive for PM1, PM2, PM3, PP2, PP3, and PP4), and the p.(Leu104Val) was assessed as "pathogenic" (positive for PS1, PM1, PM2, PP2, PP3, PP4, and PP5) (for details, see Richards et al. [8]). While WES also identified several rare variants (minor allele frequencies of $\leq 0.01$ in the public and in-house databases utilized in this study) under the assumption of Mendelian 
Table 1 Blood endocrine data of this patient

\begin{tabular}{|c|c|c|c|c|}
\hline & \multicolumn{2}{|c|}{ Patient } & \multicolumn{2}{|c|}{ Reference value $e^{\S}$} \\
\hline & Basal* & Peak $^{\dagger}$ & Basal & Peak \\
\hline \multicolumn{5}{|l|}{$<1110 / 12$ years of age $>$} \\
\hline $\mathrm{GH}(\mathrm{ng} / \mathrm{mL})$ & 0.19 & 11.8 & $<3.0$ & $>6.0$ \\
\hline ACTH $(\mathrm{pg} / \mathrm{mL})$ & 58 & 88 & $22.0 \pm 16.8$ & $>50$ \\
\hline Cortisol $(\mu \mathrm{g} / \mathrm{dL})$ & 16.3 & 19.9 & $3.0-12.0$ & \\
\hline LH (mIU/mL) & 19.3 & 112.5 & $0.1-0.4^{\pi}$ & $1.6-4.8^{\pi}$ \\
\hline $\mathrm{FSH}(\mathrm{mIU} / \mathrm{mL})$ & 105.3 & 180.3 & $2.1-6.1^{\pi}$ & $14.5-21.9$ \\
\hline $\mathrm{TSH}(\mu \mathrm{U} / \mathrm{mL})$ & 4.1 & 16.7 & $0.62-3.36$ & $10-35$ \\
\hline Prolactin (ng/mL) & 22.8 & 140.8 & $1.2-12.9$ & $<70$ \\
\hline Glucose $(\mathrm{mg} / \mathrm{dL})$ & 80 & 60 & $60-100$ & \\
\hline Insulin (IRI) $(\mu \mathrm{U} / \mathrm{mL})$ & 2 & & $2-10$ & \\
\hline $\mathrm{HbA1c}(\%)$ & 5.3 & & $4.6-6.2$ & \\
\hline IGF-I (ng/mL) & $145^{\ddagger}$ & & $155-588$ & \\
\hline Estradiol $(\mathrm{pg} / \mathrm{mL})$ & $<2.5$ & & $0-63$ & \\
\hline Free T4 (ng/dL) & 1.14 & & $0.98-1.90$ & \\
\hline $\operatorname{Tg}(\mathrm{ng} / \mathrm{mL})$ & 104.5 & & $10-30$ & \\
\hline Anti-TPO-antibody & Negative & & Negative & \\
\hline Anti-Tg-antibody & Negative & & Negative & \\
\hline \multicolumn{5}{|l|}{$<191 / 12$ years of age $>$} \\
\hline $\mathrm{TSH}(\mu \mathrm{U} / \mathrm{mL})$ & 1.68 & & $0.62-3.36$ & \\
\hline Free $\mathrm{T}_{4}(\mathrm{ng} / \mathrm{dL})$ & $\underline{0.88}$ & & $0.98-1.90$ & \\
\hline $\operatorname{Tg}(\mathrm{ng} / \mathrm{mL})$ & 466.4 & & $10-30$ & \\
\hline \multicolumn{5}{|l|}{$<238 / 12$ years of age $>$} \\
\hline $\mathrm{AMH}(\mathrm{ng} / \mathrm{mL})$ & $\leq 0.01$ & & $2.00-12.50$ & \\
\hline
\end{tabular}

Abbreviations: IRI, immunoreactive insulin; Tg, thyroglobulin; TPO, thyroid peroxidase; and AMH, anti-Müllerian hormone.

The data above and below the reference values are boldfaced and underlined, respectively.

* Basal values obtained at 9:00 am in a fasting condition.

${ }^{\dagger}$ The highest or lowest values during a triple tolerance test (insulin $0.1 \mathrm{U} / \mathrm{kg}, \mathrm{GnRH} 100 \mu \mathrm{g} / \mathrm{m}^{2}$, and TRH $300 \mu \mathrm{g} / \mathrm{m}^{2}$ bolus i.v.; blood sampling: 0, 30, 60, 90, and $120 \mathrm{~min}$ ).

$\$$ Although the IGF-1 value is lower than the age-matched female reference range, it appears to be normal for prepubertal girls.

$\S$ Age- and sex-matched Japanese reference values.

" Reference ranges in prepubertal girls.

inheritance with complete penetrance (Table 2), there was no data in support of the relevance of such variants to the phenotypic development of this patient, in terms of pathogenicity and known phenotype.

\section{N-glycosylation Studies}

The N-glycosylation status of transferrin was examined by the electrospray ionization mass spectrometry (ESI-MS) analysis, as described previously [9]. In brief, transferrin was purified from serum by immunoaffinity with rabbit polyclonal antibody against human transferrin, and was subjected to liquid chromatography-ESI-MS using a reversed-phase (C4) minicolumn and an
API4500 quadrupole mass spectrometer (Sciex). The obtained mass spectrum of multiply-charged ions was transformed into a single-charge spectrum using a Promass protein deconvolution software (ThermoFisher Scientific).

Consequently, asialotransferrin and disialotransferrin compatible with PMM2-CDG were identified in this patient, together with the normally glycosylated tetrasialotransferrin (Fig. 2B). Such aberrant transferrins were barely detected in a control subject, while tetrasialotransferrin was clearly delineated. 


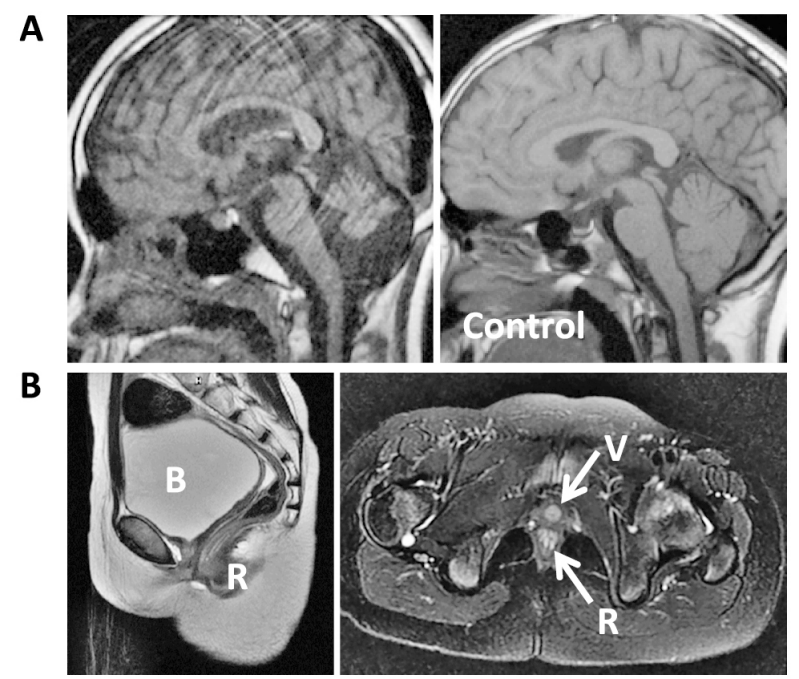

Fig. 1 MRI findings.

A. Brain MRI showing cerebellar hypotrophy.

B. Pelvic MRI delineating a rudimentary uterus and an apparent lack of recognizable ovaries. B, bladder; R, rectum; and $\mathrm{V}$, vagina.

\section{Discussion}

The present study indicates that this patient had PMM2-CDG. Indeed, molecular studies identified biallelic mutations in $P M M 2$ which were extremely rare in the general populations and were assessed to be pathogenic. While the p.(Asp12His) has not been reported previously, the p.(Leu104Val) has been shown to be a hypomorphic mutation by the previous functional study [10]. Furthermore, N-glycosylation studies revealed asialotransferrin and disialotransferrin compatible with an impaired biosynthesis of lipid-linked oligosaccharide in PMM2-CDG [9]. The presence of normally glycosylated tetrasialotransferrin would reflect the residual activity of the mutant PMM2 proteins, because complete lack of PMM2 function has been assumed to be lethal [4]. Consistent with such genetic and biochemical findings, this patient exhibited multiple clinical features reported in PMM2-CDG, such as characteristic face, intellectual disability, cerebellar hypotrophy, skeletal abnormalities, and coagulation abnormalities.

This patient also had POI which is almost invariably present in pubertal to adult females with PMM2-CDG [5, 6]. POI in PMM2-CDG has been assumed to be due to apparently hypergonadotropic but functionally hypogonadotropic hypogonadism because of compromised bioactivity of FSH/LH (especially FSH) with glycosylation defects [11, 12]. However, FSH bioactivity has been shown to remain at a low-normal range in PMM2-CGD $[11,13]$. Furthermore, since AMH was undetectable in this patient, this suggests the occurrence of folliculogenic failure at an early stage before the FSH-independent development of AMH-producing preantral and small antral follicles rather than the FSH-dependent subsequent development of non-AMH-producing antral and preovulatory follicles [14]. Indeed, serum AMH values remain grossly normal or rather increased in patients with hypogonadotropic hypogonadism $[15,16]$. In this regard, it is notable that a critical role of glycosylation in oocyte development and function has been suggested: (1) PMM2 is strongly expressed in the cytoplasm of oocytes in a human fetus aborted at 25 weeks of gestation [17]; (2) many proteins expressed in oocytes and involved in ovarian development and function such as BMP15 and GDF9 are glycosylated [18, 19]; (3) mice lacking Clgakt1 (core 1 synthase, glycoprotein-N-acetylgalactosamine 3$\beta$-galactosyltransferase 1) and Mgat1 ( $\alpha$-1,3-mannosylglycoprotein $2-\beta-\mathrm{N}$-acetylglucosaminyltransferase) exhibit POI because of deficiency of core 1-derived O-glycans and complex N-glycans in oocytes [20]; and (4) oocytespecific deletion of Mgat1 leads to defective preovulatory follicles in mice [21]. Collectively, it is inferred that PMM2 mutations and resultant $\mathrm{N}$-glycosylation defects lead to syndromic POI primarily by affecting oogenesis and/or oocyte-dependent early folliculogenesis rather than by impairing gonadotropin functions.

Although ovaries were undetected by MRI in this patient, it is unlikely that $P M M 2$ mutations cause ovarian agenesis. Ovarian (gonadal) agenesis is caused by impaired formation of an indifferent gonad common to both sexes, in contrast to subsequent male-specific testis development with hormone-producing somatic cells or female-specific ovarian development with meiotic oocytes [22]. Since androgen-dependent male sex differentiation is preserved in PMM2-CDG [5, 6], this suggests the development of an indifferent gonad (at least somatic portion of the indifferent gonad) and subsequent hormone-producing testis formation in male patients with PMM2-CDG. Thus, it is inferred that this patient had rudimentary streak-like dysgenetic ovaries rather than ovarian agenesis, although ovarian regression may be possible [13]. In this context, since pubertal to adult males with PMM2-CDG have small testes (usually $\leq 5$ $\mathrm{mL}$ ) indicative of spermatogenic failure [11, 12], this may imply that $P M M 2$ is involved in meiosis common to both sexes.

The mother with the heterozygous PMM2 mutation had no clinical finding suggestive of ovarian dysfunction. Consistent with this, there is no report describing ovarian dysfunction in females heterozygous for PMM2 mutations, although no description might not necessarily indicate lack of ovarian dysfunction in such females. It is likely, therefore, that $P M M 2$ heterozygosity permits normal ovarian function. 


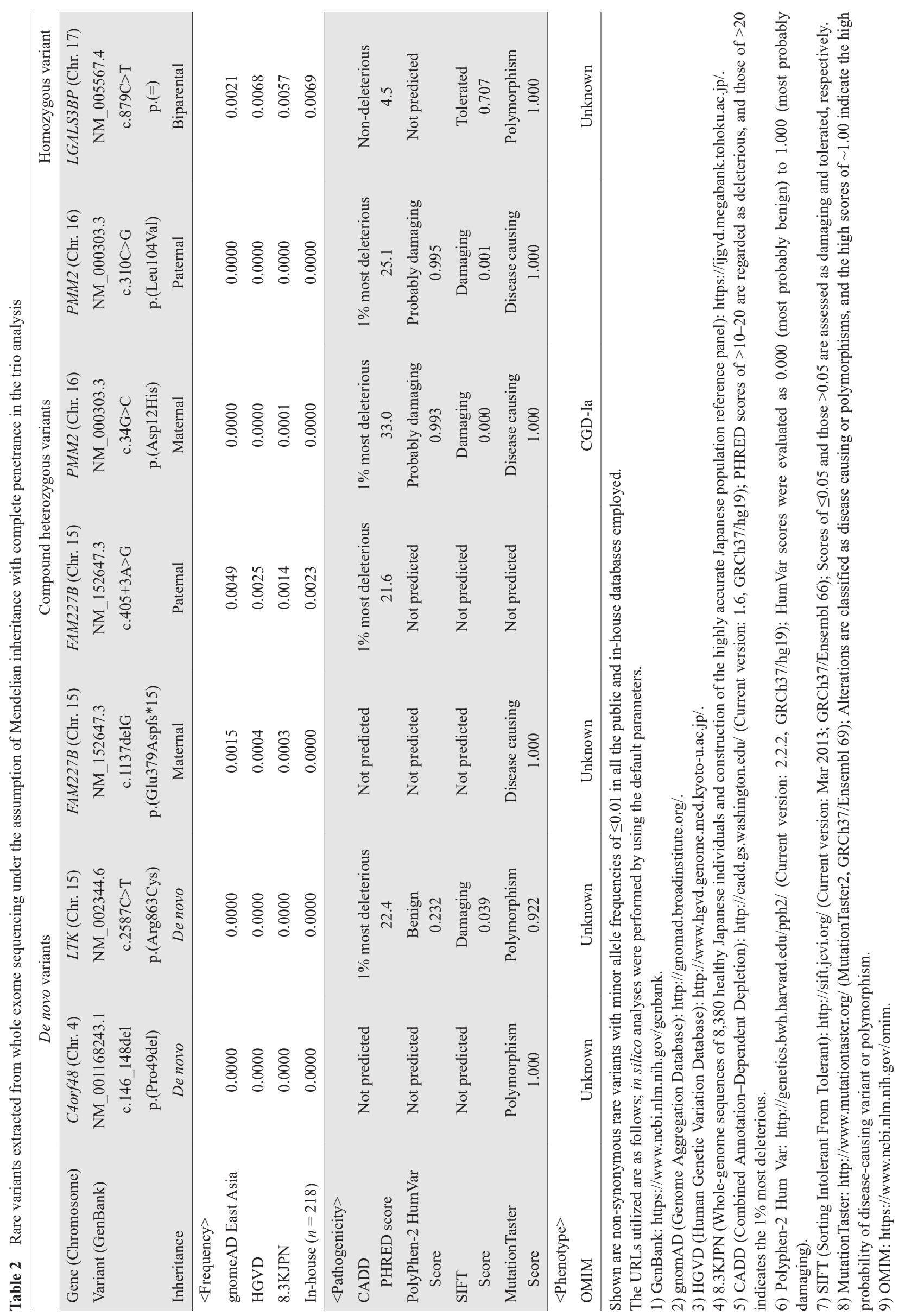


A
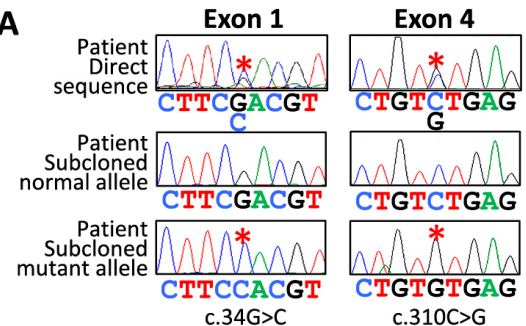

B

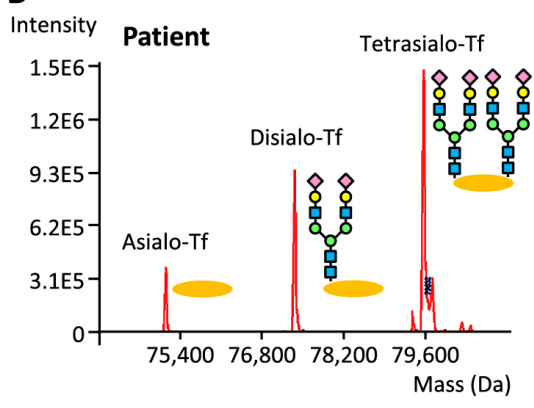

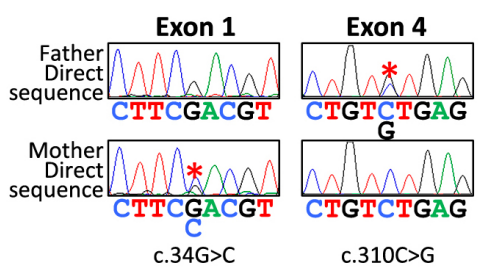

c. $34 \mathrm{G}>\mathrm{C}$

c.310C $>\mathrm{G}$

$\diamond$ Sialic acid $\mathrm{O}$ Galactose $\square \mathrm{N}$-acethylglucosamine $\mathrm{O}$ Mannose $\rightleftharpoons$ Transferrin (Tf)

Fig. 2 The results of molecular and N-glycosylation studies.

A. Electrochromatograms showing biallelic PMM2 mutations. The primers for c.34G $>\mathrm{C}$ on exon 1 are: forward, 5'AACGGAGTCCCCTCCTCTT-3'; and reverse, 5'-AGAGTTCTTCAGCCCCGTCT-3'. The primers for c.310C $>$ G on exon 4 are: forward, 5'-GACCCTGGGTTTGCTATGAA-3'; and reverse, 5'-ACTGAACCCTGCTGTGAACC-3'.

B. ESI-MS findings (deconvoluted spectrum) showing N-glycosylation status of transferrin.

PMM2-CDG is occasionally associated with several endocrine features other than POI, such as hypothyroidism, hyperprolactinemia, hypocortisolism, and hypoglycemia $[5,6,11,12,23]$. In this regard, this patient manifested antibody-negative mild hypothyroidism in the presence of overt hyperthyroglobulinemia and normal serum TSH level. Since thyroglobulin is heavily Nglycosylated [24], PMM2 mutations may compromise thyroglobulin functions, leading to hypothyroidism in patients with high susceptibility to hypothyroidism. In this context, hyperthyroglobulinemia may suggest compensatory phenomenon against reduced thyroglobulin functions, and normal serum TSH level would be explained by the mild degree of hypothyroidism. This patient also exhibited mild hyperprolactinemia. Since her thyroid function was normal at the time of prolactin measurement, it is unlikely that hyperprolactinemia is due to increased TRH secretion resulting from hypothyroidism. Thus, our data would support the notion that the decreased dopamine action may underlie the development of hyperprolactinemia in a certain fraction of patients [11].

In summary, we revealed PMM2-CDG in a female with syndromic POI. The results, in conjunction with the previous findings [1], imply that $P M M 2$ constitutes an important gene for syndromic POI. In this regard, while many clinical features such as characteristic face, growth failure, and intellectual disability are non-specific, cerebellar ataxia is a fairly characteristic and highly prevalent feature in PMM2-CDG [5, 6]. Thus, it is recommended to examine $P M M 2$ in patients with syndromic POI, especially those with cerebellar ataxia/hypotrophy. In addition, since homozygous PMM2 p.Gly186Arg mutation with a relatively high residual activity has been identified in sisters with apparently non-syndromic POI [17], $P M M 2$ may also be worth examining in patients with non-syndromic POI. Further studies will permit to clarify the relevance of PMM2-CDG to the development of syndromic and non-syndromic POI.

\section{Acknowledgements}

We thank Ms. Aya Kitamoto for her technical support.

\section{Disclosure}

The authors declare no conflict of interest.

\section{Financial Support}

This study was funded by Grants-in-Aid for Scientific Research (A) (JP20H00539) and (C) (19K10658), and for Scientific Research on Innovative Areas (JP17H06428) from the Japan Society for the Promotion 
of Science (JSPS), and by Grants from the Japan Agency for Medical Research and Development (AMED)
(JP20ek0109301).

\section{References}

1. Tucker EJ, Grover SR, Bachelot A, Touraine P, Sinclair AH (2016) Premature ovarian insufficiency: new perspectives on genetic cause and phenotypic spectrum. Endocr Rev 37: 609-635.

2. França MM, Mendonca BB (2019) Genetics of primary ovarian insufficiency in the next-generation sequencing era. J Endocr Soc 4: bvz037.

3. Freeze HH, Chong JX, Bamshad MJ, Ng BG (2014) Solving glycosylation disorders: fundamental approaches reveal complicated pathways. Am J Hum Genet 94: 161175.

4. Matthijs G, Schollen E, Bjursell C, Erlandson A, Freeze $\mathrm{H}$, et al. (2000) Mutations in PMM2 that cause congenital disorders of glycosylation, type Ia (CDG-Ia). Hum Mutat 16: 386-394.

5. Monin ML, Mignot C, De Lonlay P, Héron B, Masurel A, et al. (2014) 29 French adult patients with PMM2congenital disorder of glycosylation: outcome of the classical pediatric phenotype and depiction of a late-onset phenotype. Orphanet J Rare Dis 9: 207.

6. Schiff M, Roda C, Monin ML, Arion A, Barth M, et al. (2017) Clinical, laboratory and molecular findings and long-term follow-up data in 96 French patients with PMM2-CDG (phosphomannomutase 2-congenital disorder of glycosylation) and review of the literature. $\mathrm{J} \mathrm{Med}$ Genet 54: 843-851.

7. Miyado M, Fukami M, Takada S, Terao M, Nakabayashi $\mathrm{K}$, et al. (2019) Germline-derived gain-of-function variants of Gs $\alpha$-coding GNAS gene identified in nephrogenic syndrome of inappropriate antidiuresis. J Am Soc Nephrol 30: 877-889.

8. Richards S, Aziz N, Bale S, Bick D, Das S, et al. (2015) ACMG Laboratory Quality Assurance Committee. Standards and guidelines for the interpretation of sequence variants: a joint consensus recommendation of the American college of medical genetics and genomics and the association for molecular pathology. Genet Med 17: 405-424.

9. Wada Y (2016) Mass spectrometry of transferrin glycoforms to detect congenital disorders of glycosylation: sitespecific profiles and pitfalls. Proteomics 16: 3105-3110.

10. Westphal V, Enns GM, McCracken MF, Freeze HH (2001) Functional analysis of novel mutations in a congenital disorder of glycosylation Ia patient with mixed Asian ancestry. Mol Genet Metab 73: 71-76.

11. de Zegher F, Jaeken J (1995) Endocrinology of the carbohydrate-deficient glycoprotein syndrome type 1 from birth through adolescence. Pediatr Res 37: 395-401.

12. Miller BS, Freeze HH (2003) New disorders in carbohydrate metabolism: congenital disorders of glycosylation and their impact on the endocrine system. Rev Endocr Metab Disord 4: 103-113.
13. Kristiansson B, Stibler H, Wide L (1995) Gonadal function and glycoprotein hormones in the carbohydratedeficient glycoprotein (CDG) syndrome. Acta Paediatr 84: 655-659.

14. Moolhuijsen LME, Visser JA (2020) Anti-Mullerian hormone and ovarian reserve: update on assessing ovarian function. J Clin Endocrinol Metab 105: 3361-3373.

15. Bry-Gauillard H, Larrat-Ledoux F, Levaillant JM, Massin N, Maione L (2017) Anti-Müllerian hormone and ovarian morphology in women with isolated hypogonadotropic hypogonadism/Kallmann syndrome: effects of recombinant human FSH. J Clin Endocrinol Metab 102: 11021111.

16. Cecchino GN, Canillas GM, Cruz M, García-Velasco JA (2019) Impact of hypogonadotropic hypogonadism on ovarian reserve and response. J Assist Reprod Genet 36: 2379-2384.

17. Peng T, Lv C, Tan H, Huang J, He H, et al. (2020) Novel PMM2 missense mutation in a Chinese family with nonsyndromic premature ovarian insufficiency. $J$ Assist Reprod Genet 37: 443-450.

18. Dube JL, Wang P, Elvin J, Lyons KM, Celeste AJ, et al. (1998) The bone morphogenetic protein 15 gene is Xlinked and expressed in oocytes. Mol Endocrinol 12: 1809-1817.

19. Hayashi M, McGee E, Min G, Klein C, Rose UM, et al. (1999) Recombinant growth differentiation factor-9 (GDF-9) enhances growth and differentiation of cultured early ovarian follicles. Endocrinology 140: 1236-1244.

20. Williams SA, Stanley P (2011) Premature ovarian failure in mice with oocytes lacking core 1-derived 0-glycans and complex N-glycans. Endocrinology 152: 1057-1066.

21. Williams SA, Stanley P (2009) Oocyte-specific deletion of complex and hybrid $\mathrm{N}$-glycans leads to defects in preovulatory follicle and cumulus mass development. Reproduction 137: 321-331.

22. Achermann JC, Hughes IA (2016) Pediatric disorders of sex development. In: Melmed S, Polonsky KS, Larsen PR, Kronrnberg HM (eds) Williams textbook of Endocrinology (13th). Elsevier, Philadelphia, USA: 893-963.

23. Shanti B, Silink M, Bhattacharya K, Howard NJ, Carpenter K, et al. (2009) Congenital disorder of glycosylation type Ia: heterogeneity in the clinical presentation from multivisceral failure to hyperinsulinaemic hypoglycaemia as leading symptoms in three infants with phosphomannomutase deficiency. J Inherit Metab Dis 32 Suppl 1: S241-S251.

24. Ząbczyńska M, Kozłowska K, Pocheć E (2018) Glycosylation in the thyroid gland: vital aspects of glycoprotein function in thyrocyte physiology and thyroid disorders. Int J Mol Sci 19: 2792. 